\title{
Blockade of DNA methylation enhances the therapeutic effect of gefitinib in non-small cell lung cancer cells
}

\author{
XIAO-YOU LI, JIAN-ZHONG WU, HAI-XIA CAO, RONG MA, \\ JIAN-QIU WU, YUE-JIAO ZHONG and JI-FENG FENG
}

Department of Chemotherapy, Jiangsu Cancer Hospital and Research Institute, Nanjing, Jiangsu 210009, P.R. China

Received September 25, 2012; Accepted December 7, 2012

DOI: 10.3892/or.2013.2298

\begin{abstract}
The sensitivity of lung cancer to epidermal growth factor receptor (EGFR)-tyrosine kinase inhibitors (TKIs) has been found to be associated with mutations in the tyrosine kinase domain of EGFR. However, not all mutations are sensitive to gefitinib. While $\mathrm{CpG}$ island methylation in the promoter region of the EGFR gene and transcriptional silencing are common in solid tumors, the role of the EGFR gene promoter methylation in affecting resistance to TKIs in non-small cell lung cancer (NSCLC) remains unknown. In this study, we examined the correlation between EGFR gene promoter methylation and the therapeutic effect of gefitinib in NSCLC cells. Three NSCLC cell lines with different EGFR mutation statuses and levels of sensitivity to EGFR-TKIs were used in this study: H1650 (del E746-A750), H1299 (wild-type EGFR) and PC-9 (del E746-A750). Cells were treated with gefitinib or 5-aza-2'-deoxy cytidine (5-aza-CdR), a methylation inhibitor, alone or in combination. Subsequently, the methylation status of the EGFR gene promoter was examined by methylation-specific PCR (MSP). Cell survival and apoptosis assays were performed using the Cell Counting Kit-8 (CCK-8) and flow cytometry. In addition, western blot analysis and quantitative real-time PCR were used to examine the expression levels of EGFR protein and mRNA. Our study showed that the promoter region of the EGFR gene in PC-9 cells was unmethylated, and that the cells were sensitive to gefitinib. By contrast, the promoter region of the EGFR gene in the H1650 and H1299 cells was methylated, and the cells were resistant to gefitinib. Of note, the combination treatment with 5-aza-CdR and gefitinib further enhanced the growth inhibitory effects and led to the induction of apoptosis, while a significant reduction in the expression of EGFR protein and mRNA was observed in the H1650 and H1299 cells. These results suggest that blockade of DNA methylation may enhance the antitumor effects of EGFR-TKIs and gefitinib in NSCLC
\end{abstract}

Correspondence to: Dr Ji-Feng Feng, Department of Chemotherapy, Jiangsu Cancer Hospital and Research Institute, 42 Baizi Ting, Xuanwu, Nanjing, Jiangsu 210009, P.R. China

E-mail: fjif@vip.sina.com

Key words: epidermal growth factor receptor, tyrosine kinase inhibitor, non-small cell lung cancer, methylation, gefitinib, 5-aza-2'-deoxycytidine cells. Thus, EGFR gene promoter methylation may be a potential mechanism for acquired resistance to gefitinib.

\section{Introduction}

Lung cancer is the most lethal malignancy worldwide. Treatment options remain limited for some patients with advanced lung cancer. Approximately half of limited stage lung cancer patients relapse despite curative intent surgery/radiation and adjuvant chemotherapy (1). Thus, there is an urgent need to develop novel or modified therapeutic approaches to enhance the efficacy of treatment for this malignancy.

Epidermal growth factor receptor (EGFR), a $170 \mathrm{kDa}$ membrane-bound protein encoded by 28 exons on chromosome $7 \mathrm{p} 12$, is a typical member of the tyrosine kinase (TK) family and belongs to a subfamily that consists of four closely related members: EGFR (ErbB1), HER-2/neu (ErbB2), HER-3 (ErbB3) and HER-4 (ErbB4). All members have an extracellular ligandbinding domain, a single membrane spanning domain and an intracellular domain $(2,3)$; they are also known to have intrinsic TK activity apart from ErbB3 (4). EGFR has been shown to play a central role in the occurrence and progression of multiple solid tumors, including lung cancer (5). Several anti-EGFR molecules have been reported to induce neoplastic growth inhibition. Among these, gefitinib (Iressa), an orally available synthetic anilinoquinazoline agent, selectively binds to the TK region of the intracellular domain of EGFR, prevents ATP binding, and blocks EGFR signaling transduction pathways, thereby inhibiting cancer cell growth (6). Over the years, non-small cell lung cancer (NSCLC) containing EGFR mutations has been shown to be sensitive to gefitinib, and this agent has been successfully used in the treatment of these patients $(7,8)$. Unfortunately, some patients with EGFR gene mutations develop resistance to TK inhibitor (TKI) treatment after a certain period of time, and the mechanisms behind this resistance remain unknown.

Aberrant hypermethylation in the gene promoter has become one of the major mechanisms for silencing tumor suppressor or other cancer-associated genes in many types of human cancer, and it is an epigenetic modification that plays an important role in the control of gene expression in mammalian cells (9). Recently, the role it plays in carcinogenesis has raised considerable interest (10). In many tumor types, $\mathrm{CpG}$ island hypermethylation in the promoter region of several tumor suppressor genes has been observed and has been shown to 
correlate closely with the loss of mRNA and protein expression. While hypermethylation typically affects tumor suppressor genes, it also silences oncogenes, such as cyclooxygenase-2 (COX-2) (11) and telomerase reverse transcriptase (TERT) (12). Since the promoter region of the EGFR gene contains a CpG island that extends into exon 1, we hypothesized that EGFR gene promoter methylation may influence the antitumor effect of gefitinib on NSCLC cells.

\section{Materials and methods}

Cell culture and reagents. Three NSCLC cell lines with different EGFR mutation statuses and levels of sensitivity to EGFR-TKIs, obtained from the Shanghai Cell Station, Chinese Academy of Sciences, Shanghai, China were used: H1650 (del E746-A750), H1299 (wild-type EGFR) and PC-9 (del E746A750). These cells were maintained in RPMI-1640 culture medium containing $10 \%$ heat-inactivated fetal bovine serum (FBS), $100 \mathrm{U} / \mathrm{ml}$ penicillin and $100 \mathrm{mg} / \mathrm{ml}$ streptomycin, and they were incubated in humidified air and $5 \% \mathrm{CO}_{2}$ at $37^{\circ} \mathrm{C}$. Gefitinib was a generous gift from AstraZeneca (Cheshire, UK). Stock solutions were prepared in dimethyl sulfoxide (DMSO) and stored at $-20^{\circ} \mathrm{C}$. Gefitinib solution was prepared in fresh medium prior to each experiment and the control cells were treated with medium containing an equal concentration of DMSO. 5-Aza-deoxycytidine (5-aza-CdR, decitabine), a methylation inhibitor, was purchased from Sigma (St. Louis, MO, USA) and prepared as described above.

Methylation-specific PCR (MSP). For the demethylation experiments, NSCLC cells (H1650 and H1299) were treated with varying concentrations $(1-10 \mu \mathrm{M})$ of 5-aza-CdR for up to $72 \mathrm{~h}$. The PC-9 cells were not treated with 5-aza-CdR due to their unmethylated status. Genomic DNA was extracted by proteinase $\mathrm{K}$ digestion followed by purification with a series of phenol/chloroform and isopropyl alcohol precipitations. The extracted DNA samples were stored in TE buffer at $-20^{\circ} \mathrm{C}$ until use. Bisulfite-based DNA modification, which converted all unmethylated cytosines to uracil, was performed by using the Methylcode Bisulfite Conversion kit (Invitrogen, Carlsbad, CA, USA) according to the manufacturer's instructions. The modified DNA was used as a template for MSP with primers specific for either the modified-methylated or unmethylated EGFR gene promoter sequences. PCR amplification was performed with the following primer sets that included the $\mathrm{CpG}$ island of EGFR: forward primer, 5'-GGTTGGGTTTGTAAGTTCGC-3' and reverse primer, 5'-ATAAACAACGATAACCCCCG-3' for the methylated EGFR sequence (150 bp); and forward primer, 5'-GGTTGGGTTTGTAAGTTTGT-3' and reverse primer, 5'-ATAAACAACAATAACCCCCA-3' for the unmethylated EGFR sequence (150 bp). The PCR amplification program consisted of $10 \mathrm{~min}$ at $95^{\circ} \mathrm{C}$, followed by 40 cycles of $30 \mathrm{sec}$ denaturation at $95^{\circ} \mathrm{C}, 30 \mathrm{sec}$ of annealing at $56^{\circ} \mathrm{C}, 30 \mathrm{sec}$ of extension at $72^{\circ} \mathrm{C}$, with a final extension at $72^{\circ} \mathrm{C}$ for $10 \mathrm{~min}$ for both primers using HotStar Taq DNA polymerase (Qiagen, Valencia, CA, USA). PCR products were separated on $3 \%$ agarose gels with ethidium bromide and visualized under UV illumination. As the positive control, M.SssI methylase (New England BioLabs, Ipswich, MA, USA) was used to methylate normal human peripheral blood.
Cell proliferation assays. Cell proliferation assays were performed using a Cell Counting Kit-8 (CCK-8) (Dojindo Molecular Technologies, Inc., Kumamoto, Japan) according to the manufacturer's instructions. Briefly, the H1650, H1299 and PC-9 cells were seeded at a density of 3,000-5,000 cells/ well in 96-well microtiter plates for $24 \mathrm{~h}$. While the H1650 and H1299 cells were treated with increasing concentrations of gefitinib (0.01-100 $\mu \mathrm{M}), 5$-aza-CdR $(0.01-100 \mu \mathrm{M})$, or a combination of gefitinib and 5-aza-CdR for up to $72 \mathrm{~h}$, the PC-9 cells were treated with increasing concentrations of gefitinib (0.01-100 $\mu \mathrm{M})$ alone for $72 \mathrm{~h}$. Subsequently, $10 \mu \mathrm{l}$ of the CCK-8 solution were added to each well in the plate. The absorbance (A) at $450 \mathrm{~nm}$ was measured using a microplate reader, and a calibration curve was prepared using the data obtained from the wells that contained known numbers of viable cells. Each experiment was carried out in five replicate wells at each drug concentration and repeated at least three times.

Apoptosis measurements. The apoptosis assay was conducted using Annexin V staining and flow cytometry assays. The H1650 and H1299 cells seeded in 6-well plates were treated with gefitinib $(1 \mu \mathrm{M}), 5$-aza-CdR $(1 \mu \mathrm{M})$, or a combination of gefitinib and 5-aza-CdR based on their sensitivity to various drugs for 72 h. PC-9 cells were treated with various concentrations of gefitinib $(0.01-100 \mu \mathrm{M})$ for $72 \mathrm{~h}$. Subsequently, the treated and untreated cells were collected and washed with PBS. Annexin V staining was performed following the manufacturer's instructions (Trevigen, Inc., Gaithersburg, MD, USA). Briefly, the cells were incubated for $15 \mathrm{~min}$ at room temperature in the presence of $1 \mu \mathrm{l}$ of Annexin V-FITC, $1 \mu \mathrm{l}$ of propidium iodide, and $98 \mu \mathrm{l}$ of $1 \mathrm{X}$ binding buffer (all reagents were provided by the manufacturer). Following incubation, $400 \mu \mathrm{l}$ of $1 \mathrm{X}$ binding buffer were added to each tube, and the cells were analyzed by flow cytometry.

Western blot analysis. Cells were lysed in a buffer containing $1 \mathrm{mM}$ protease inhibitor [phenylmethylsulfonyl fluoride (PMSF)] and were cleared by centrifugation at 12,000 rpm for $10 \mathrm{~min}$. Protein concentration was determined using the BCA assay (Bio-Rad, Hercules, CA, USA). Protein $(80 \mu \mathrm{g})$ was dissolved in loading buffer, denatured by heating at $100^{\circ} \mathrm{C}$ for $5 \mathrm{~min}$, and subsequently separated on $8 \%$ polyacrylamide gels by SDS-gel electrophoresis. After separation, the proteins were transferred onto an immunoblot polyvinylidene difluoride membrane (Bio-Rad). Overall protein loading was confirmed by Ponceau S staining. Membranes were blocked with 5\% non-fat milk for $2 \mathrm{~h}$ at $37^{\circ} \mathrm{C}$ and then incubated with anti-EGFR antibody (Millipore, Billerica, MA, USA) overnight at $4^{\circ} \mathrm{C}$. The membranes were washed three times with 1X PBST and incubated with secondary antibody conjugated with peroxidase (Dako, Carpinteria, CA, USA) for $1 \mathrm{~h}$ at $37^{\circ} \mathrm{C}$. After a final wash with PBST, the membranes were developed using chemiluminescence and exposed to X-ray film. The expression of EGFR was normalized to $\beta$-actin.

RT-PCR and quantitative real-time PCR. Total RNA was extracted using TRIzol Reagent (Invitrogen) according to the manufacturer's instructions. RNA concentration was qualitatively assessed using a Nanodrop UV spectrophotometer. cDNA was generated using an OmniScript RT kit (Qiagen) according to the manufacturer's instructions. Quantitative gene 
Table I. The $\mathrm{IC}_{50}$ values of gefitinib and 5-aza-CdR in the NSCLC cell lines.

\begin{tabular}{|c|c|c|c|c|c|c|}
\hline \multirow{2}{*}{$\begin{array}{l}\text { NSCLC } \\
\text { cell line }\end{array}$} & \multirow{2}{*}{ Histology } & \multirow{2}{*}{$\begin{array}{c}\text { EGFR } \\
\text { mutation status }\end{array}$} & \multirow{2}{*}{$\begin{array}{c}\text { EGFR } \\
\text { mutation status }\end{array}$} & \multirow{2}{*}{$\begin{array}{l}\text { 5-aza-CdR } \\
\mathrm{IC}_{50}(\mu \mathrm{M})^{\mathrm{a}}\end{array}$} & \multicolumn{2}{|c|}{ Gefitinib $\mathrm{IC}_{50}(\mu \mathrm{M})$} \\
\hline & & & & & No 5-aza-CdR & 5-aza-CdR \\
\hline H1650 & $\mathrm{AD}$ & mut & Methylated & $15.91 \pm 1.42$ & $14.53 \pm 1.13$ & $2.93 \pm 0.95$ \\
\hline H1299 & $\mathrm{LC}$ & wt & Methylated & $16.69 \pm 1.64$ & $18.64 \pm 1.98$ & $3.41 \pm 1.01$ \\
\hline PC-9 & $\mathrm{AD}$ & mut & Unmethylated & & $1.42 \pm 0.73$ & \\
\hline
\end{tabular}

${ }^{\mathrm{a}} \mathrm{The} \mathrm{IC}_{50}$ value was defined as the concentration required for a $50 \%$ reduction in the absorbance calculated based on the cell viability curves. EGFR, epidermal growth factor receptor; NSCLC, non-small cell lung cancer; AD, adenocarcinoma; LC, large cell; mut, mutant; wt, wild-type; $\mathrm{IC}_{50}$, half maximal inhibitory concentration.

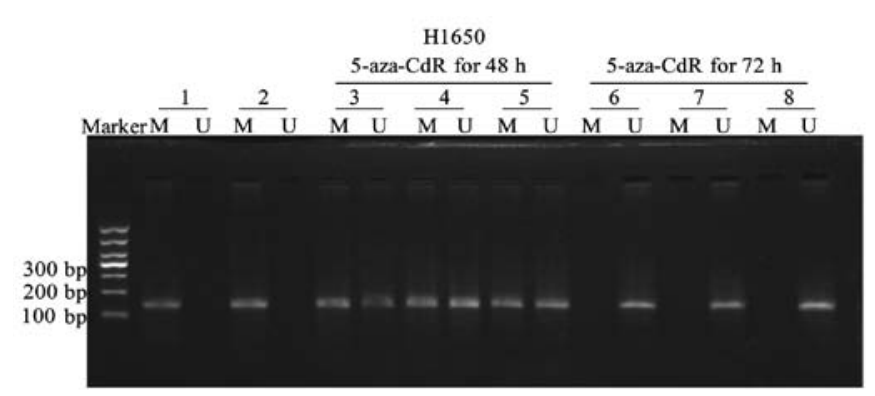

H1299
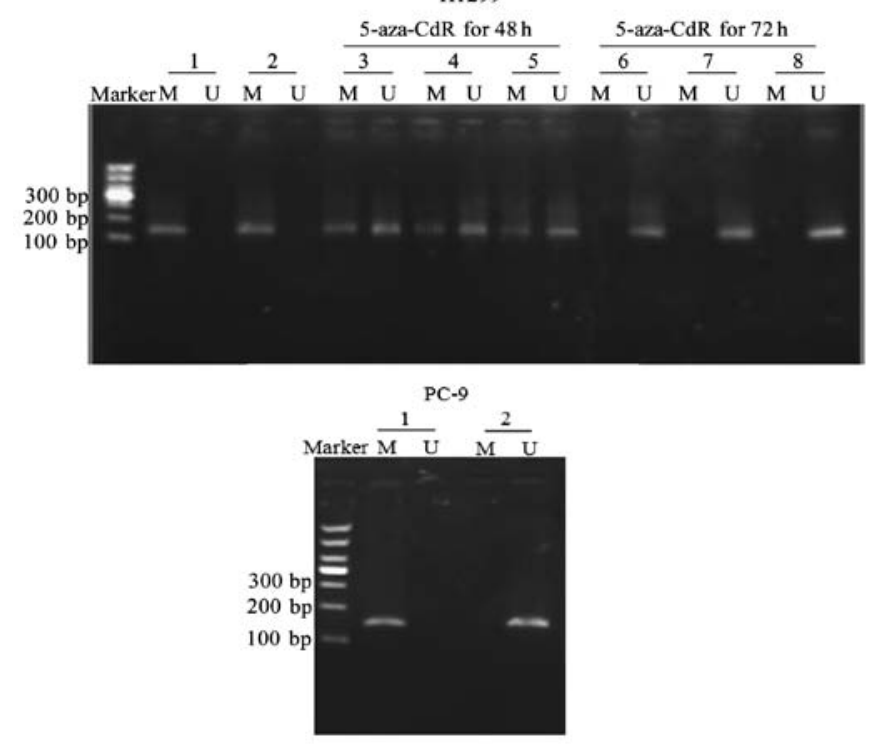

Figure 1. The $\mathrm{CpG}$ island methylation status in the promoter of the EGFR gene in NSCLC cells. NSCLC cells were treated with various concentrations (1-10 $\mu \mathrm{M})$ of 5-aza-CdR, a methylation inhibitor, for up to $72 \mathrm{~h}$. Subsequently, the cells were harvested. Genomic DNA was extracted by proteinase $\mathrm{K}$ digestion followed by purification with a series of phenol/ chloroform and isopropyl alcohol precipitations. The modified DNA was used as a template for MSP with primers specific for either the modifiedmethylated or the modified-unmethylated EGFR promoter sequences. The methylation status of the EGFR gene was determined by MSP. PCR products were separated on $3 \%$ agarose gels with ethidium bromide and visualized under UV illumination. As the positive control, M.SssI methylase was used to methylate normal human peripheral blood. Marker, DL1,000 DNA Marker; lane 1, positive control; lane 2, vehicle control; lane 3, $1 \mu \mathrm{M}$ 5-aza-CdR; lane 4, $5 \mu \mathrm{M}$ 5-aza-CdR; lane 5, $10 \mu \mathrm{M}$ 5-aza-CdR; lane 6, $1 \mu \mathrm{M}$ 5-aza-CdR; lane 7, $5 \mu \mathrm{M}$ 5-aza-CdR; lane 8, $10 \mu \mathrm{M}$ 5-aza-CdR; M, product of methylated specific primer $(150 \mathrm{bp})$; $U$, product of unmethylated specific primer (150 bp). expression analysis by reverse transcriptase-polymerase chain reaction (RT-PCR) was performed using the Universal TaqMan PCR protocol (qPCR) and the ABI 7300 sequence detection system. Briefly, $2 \mu \mathrm{l}$ of cDNA were used for each RT reaction. The $20 \mu \mathrm{l}$ PCR reaction mixture contained $1 \mathrm{X}$ primers and probe mixture (Applied Biosystems, Foster City, CA, USA). The assay IDs were as follows: Hs01076092-m1 (EGFR) and Hs99999905-m1 (GAPDH); 1X ABsolute qPCR mix (Roche, USA). The PCR conditions were $95^{\circ} \mathrm{C}$ for $10 \mathrm{~min}$, followed by 40 cycles at $95^{\circ} \mathrm{C}$ for $15 \mathrm{sec}$, and $60^{\circ} \mathrm{C}$ for $1 \mathrm{~min}$. Each sample was assayed in triplicate with commercial RNA as the positive control and RNase-free water as the negative control. Relative gene expression quantifications were calculated according to the comparative $\mathrm{Ct}$ method using GAPDH as the internal control and commercial RNA control as calibrators on each plate. The final results were determined by the formula, $2^{-\Delta \Delta C t}(13)$.

Statistical analyses. Data are expressed as the means \pm SD. Statistical analysis was performed using SPSS software version 13.0. Data were analyzed by one-way analysis of variance (ANOVA). A value of $\mathrm{P}<0.05$ was considered to indicate a statistically significant difference.

\section{Results}

$C p G$ island methylation status determination in the promoter of the EGFR gene in NSCLC cells. Three NSCLC cell lines with different EGFR gene mutation statuses were chosen in this study, including a cell line containing wild-type EGFR (H1299), a cell line containing mutant EGFR resistant to TKIs (H1650) and a cell line sensitive to TKIs (PC-9). The histological data of these NSCLC cell lines are shown in Table I. The methylation status of the EGFR gene was determined by MSP in the H1650, H1299 and PC-9 cells. As shown in Fig. 1, no methylation was observed in the PC-9 cells, while hypermethylation was detected in the H1650 and H1299 cells. We found that the EGFR gene promoter became unmethylated when the cells were treated with $1-10 \mu \mathrm{M}$ 5-aza-CdR for 48 and $72 \mathrm{~h}$ (Fig. 1).

Induction of growth inhibition by 5-aza-CdR and gefitinib. We then examined the effects of 5-aza-CdR and gefitinib on cell growth. The half maximal inhibitory concentration $\left(\mathrm{IC}_{50}\right)$ values of gefitinib in the H1650, H1299 and PC-9 cells were 14.53 \pm 1.13 , 


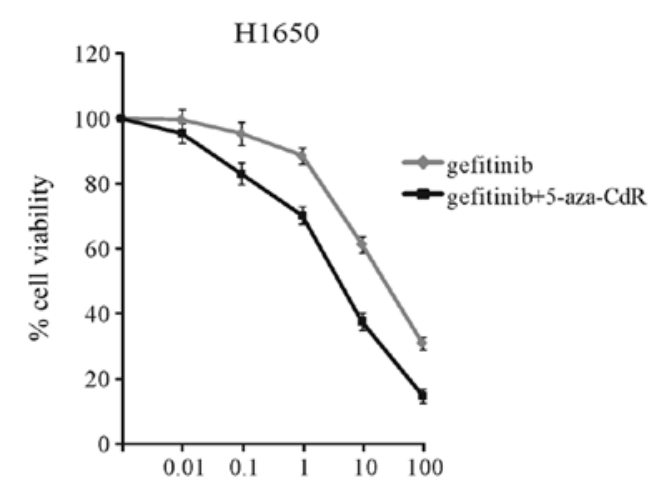

Dosage of gefitinib $(\mu \mathrm{mol} / \mathrm{l})$ for $72 \mathrm{~h}$

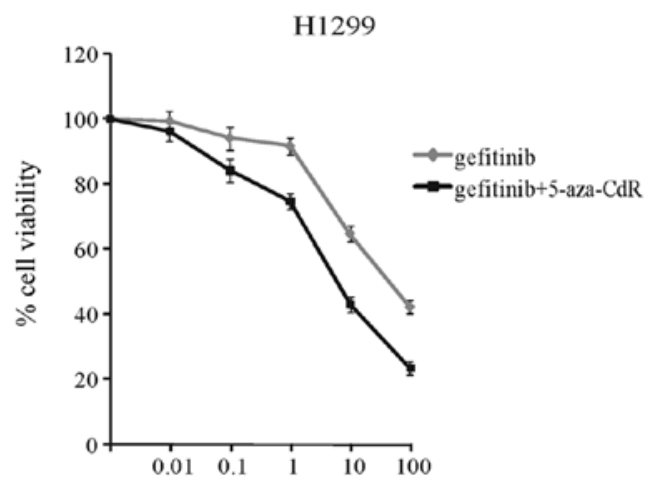

Dosage of gefitinib $(\mu \mathrm{mol} / \mathrm{l})$ for $72 \mathrm{~h}$

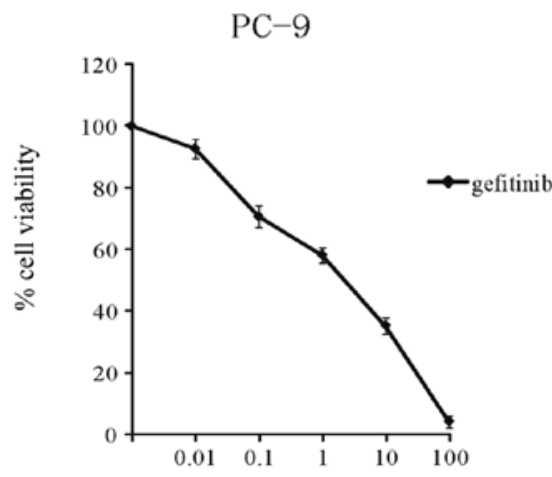

Dosage of gefitinib $(\mu \mathrm{mol} / \mathrm{l})$ for $72 \mathrm{~h}$

Figure 2. Induction of growth inhibition by 5 -aza-CdR and gefitinib. H1650 and H1299 cells were treated with increasing concentrations of gefitinib $(0.01-100 \mu \mathrm{M})$ or a combination of gefitinib $(0.01-100 \mu \mathrm{M})$ and 5-aza-CdR $(1 \mu \mathrm{M})$ for up to $72 \mathrm{~h}$. PC-9 cells were treated with increasing concentrations of gefitinib $(0.01-100 \mu \mathrm{M})$ for up to $72 \mathrm{~h}$. Subsequently, $10 \mu 1$ of the CCK-8 solution was added to each well of the plate. The absorbance (A) at $450 \mathrm{~nm}$ was measured using a microplate reader. A calibration curve was prepared using the data obtained from the wells that contained known numbers of viable cells. Each experiment was carried out in five replicate wells for each drug concentration and repeated at least three times.

$18.64 \pm 1.98$ and $1.42 \pm 0.73 \mu \mathrm{M}$, respectively. Of note, we found that in the presence of $1 \mu \mathrm{M} 5$-aza-CdR, the $\mathrm{IC}_{50}$ values of gefitinib decreased to $2.93 \pm 0.95$ and $3.41 \pm 1.01 \mu \mathrm{M}$ in the $\mathrm{H} 1650$ and H1299 cells, respectively. For the combined treatment, H1650 and H1299 cells were treated with varying concentrations (0.01-100 $\mu \mathrm{M})$ of gefitinib in the presence of $1 \mu \mathrm{M} 5$-aza-CdR; an enhanced inhibition of cell growth was observed (Fig. 2). These results suggested that 5-aza-CdR increased the cellular sensitivity of gefitinib as a cell growth inhibitor. The PC-9 cells,
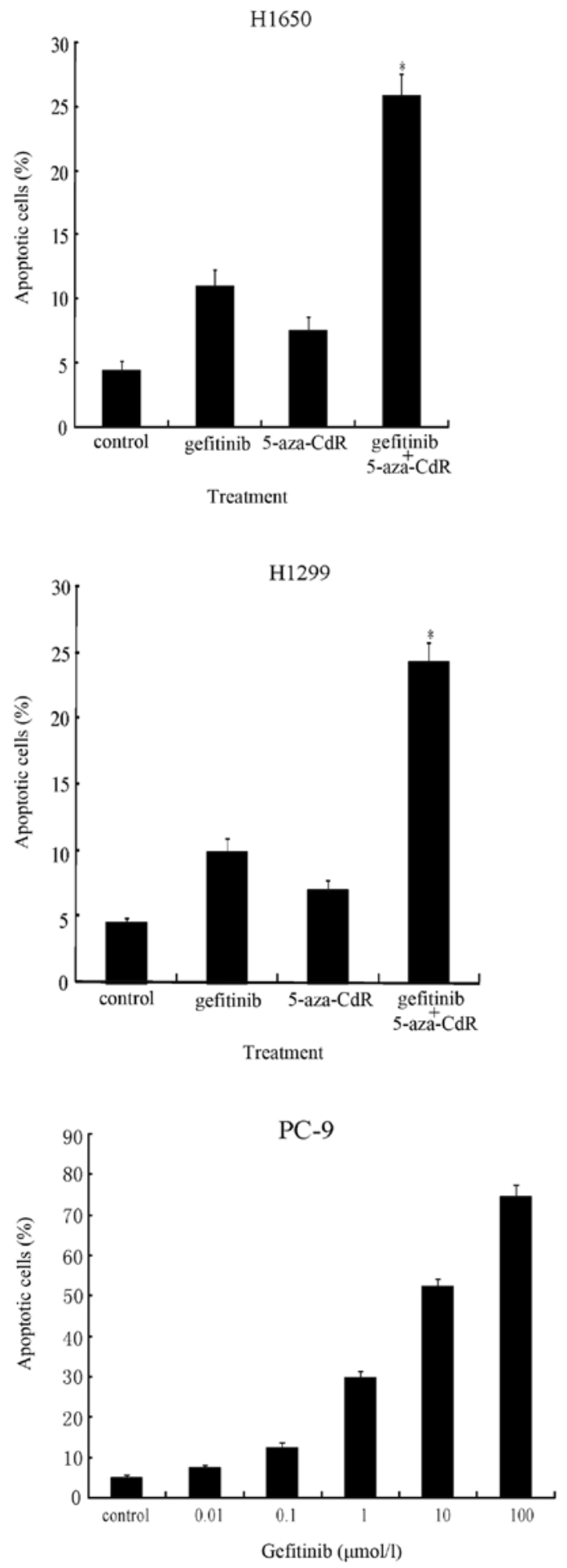

Figure 3. Induction of apoptosis by gefitinib and 5-aza-CdR. H1650 and H1299 cells seeded in 6-well plates were treated with gefitinib $(1 \mu \mathrm{M}), 5$-aza-CdR $(1 \mu \mathrm{M})$, or a combination of gefitinib and 5-aza-CdR based on their levels of sensitivity to various drugs for $72 \mathrm{~h}$. PC-9 cells were treated with increasing concentrations of gefitinib, as indicated, for up to $72 \mathrm{~h}$. Subsequently, the treated and untreated cells were collected and the apoptosis assay was conducted using Annexin V staining, followed by flow cytometry analysis according to the manufacturer's instructions. The percentage of apoptotic cells was calculated based on the instructions on the Annexin V-FITC/PI staining kit. Results are expressed as the means $\pm \mathrm{SD}$. ${ }^{*} \mathrm{P}<0.05$, compared to the control group.

which are unmethylated in the EGFR gene promoter, were more sensitive to gefitinib than the H1650 and H1299 cells (Fig. 2). 

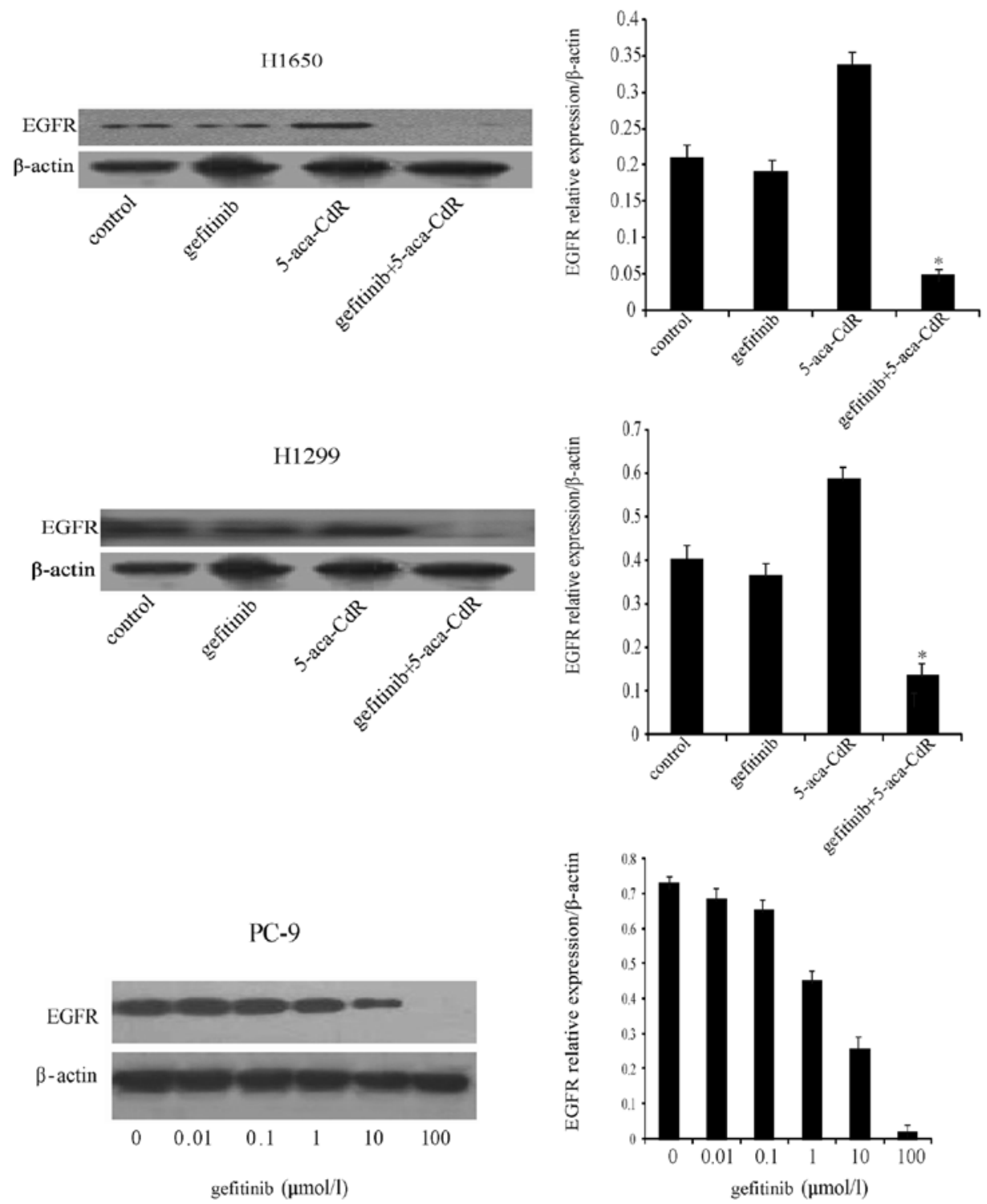

Figure 4. Effects of gefitinib and 5-aza-CdR on EGFR protein expression. H1650 and H1299 cells were treated with gefitinib $(1 \mu \mathrm{M}), 5$-aza-CdR $(1 \mu \mathrm{M})$, or a combination of gefitinib and 5-aza-CdR for up to $72 \mathrm{~h}$. In a separate experiment, PC-9 cells were treated with increasing concentrations of gefitinib, as indicated, for up to $72 \mathrm{~h}$. Subsequently, EGFR protein expression levels were determined by western blot analysis. The bar graphs indicate the densitometry results from western blot analysis. ${ }^{*} \mathrm{P}<0.05$, compared to the control group.

Induction of apoptosis by gefitinib and 5-aza-CdR. We hypothesized that a greater sensitivity to gefitinib could be achieved by EGFR demethylation in the H1650 and H1299 cells. We found that the $\mathrm{H} 1650$ and $\mathrm{H} 1299$ cell lines were relatively resistant to gefitinib with $\mathrm{IC}_{50}$ values of $14.53 \pm 1.13 \mu \mathrm{M}$ and $18.64 \pm 1.98 \mu \mathrm{M}$, respectively. Compared to either 5-aza-CdR or gefitinib treatment alone, a significant additional increase in apoptosis was observed in the $\mathrm{H} 1650$ cells $(25.73 \%, \mathrm{P}<0.05)$ and H1299 cells $(24.27 \%, \mathrm{P}<0.05)$ treated with a combination of 5-aza-CdR $(1 \mu \mathrm{M})$, which showed maximal demethylation, and gefitinib $(1 \mu \mathrm{M})$ for $72 \mathrm{~h}$ as determined by Annexin $\mathrm{V}$ staining (Fig. 3). The treatment of the unmethylated PC-9 cells with gefitinib also increased apoptosis in a dose-dependent manner (Fig. 3).

Effects of gefitinib and 5-aza-CdR on EGFR protein expression. In order to determine whether gene methylation affects protein expression, the H1650 and H1299 cells were treated with gefitinib $(1 \mu \mathrm{M}), 5$-aza-CdR $(1 \mu \mathrm{M})$, or both. As shown in Fig. 4, EGFR protein expression was low in the two cell lines. To confirm that the low expression of EGFR was due to hypermethylation, the $\mathrm{H} 1650$ and $\mathrm{H} 1299$ cells were treated with 5-aza-CdR, which resulted in $\mathrm{CpG}$ island demethylation in the EGFR gene promoter (Fig. 1). We found that EGFR protein expression was increased following treatment with 5-azaCdR. A significant decrease in EGFR protein expression was observed in the H1650 and H1299 cells after treatment with a combination of gefitinib and 5-aza-CdR compared to treatment with either agent alone. A dose-dependent reduction in EGFR protein expression was observed in the PC-9 cells treated with gefitinib (Fig. 4).

Effect of gefitinib and 5-aza-CdR on EGFR mRNA expression. We also evaluated EGFR mRNA expression by real-time PCR (qPCR) in the H1650, H1299 and PC-9 cells. Cells exposed to 5 -aza-CdR for $72 \mathrm{~h}$ demonstrated an induction of EGFR 

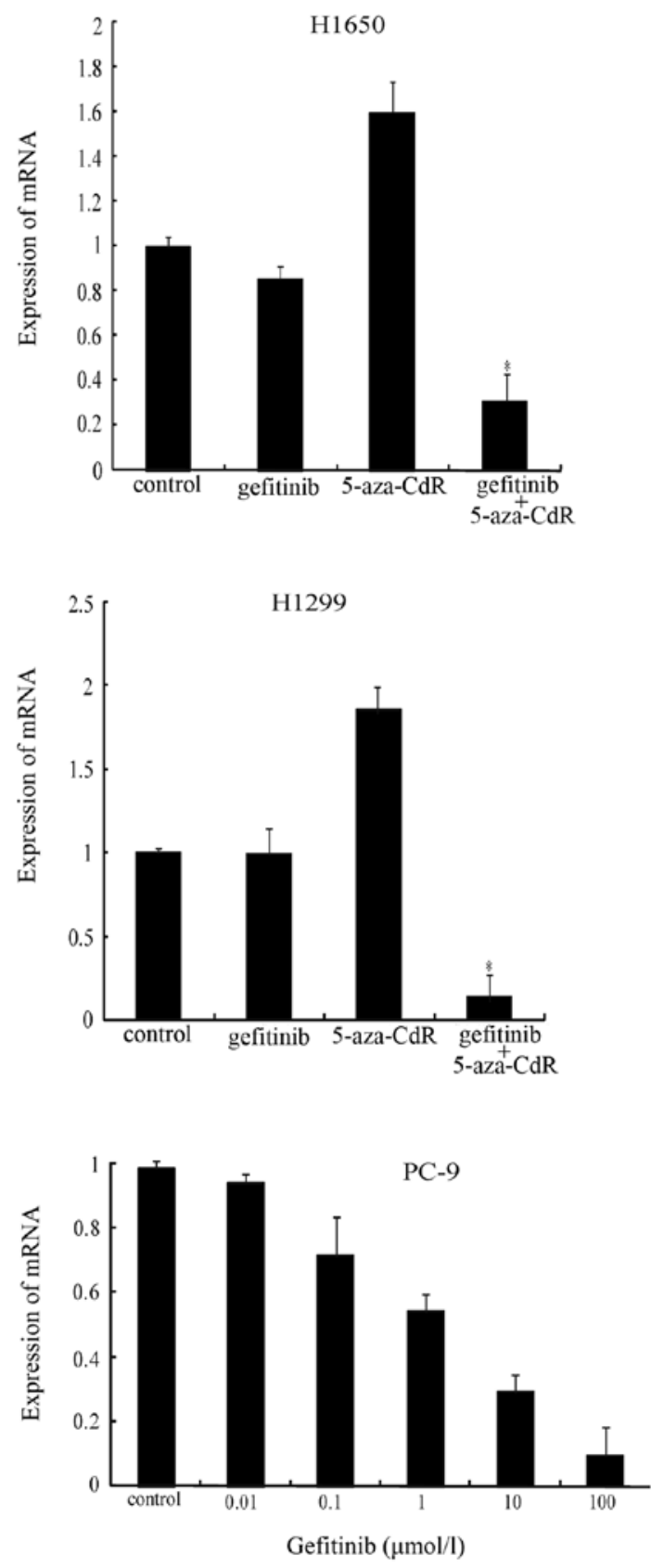

Figure 5. Effect of gefitinib and 5-aza-CdR on EGFR mRNA expression. $\mathrm{H} 1650$ and $\mathrm{H} 1299$ cells were treated with gefitinib $(1 \mu \mathrm{M}), 5$-aza-CdR $(1 \mu \mathrm{M})$, or a combination of gefitinib and 5-aza-CdR for up to $72 \mathrm{~h}$. In a separate experiment, PC-9 cells were treated with increasing concentrations of gefitinib, as indicated, for up to $72 \mathrm{~h}$. Subsequently, EGFR mRNA expression levels were determined by RT-PCR analysis. Results are presented as the means $\pm \mathrm{SD}$. ${ }^{*} \mathrm{P}<0.05$, compared to the control group.

mRNA expression (Fig. 5). The effect of the simultaneous exposure of the NSCLC cells to gefitinib in combination with 5 -aza-CdR at concentrations below the $\mathrm{IC}_{50}$ value was examined. Similar to the protein expression, further inhibition of EGFR mRNA expression was observed with the combination treatment (Fig. 5); these results indicated that the cells became more sensitive to gefitinib in the presence of 5-aza-CdR and suggested a clear synergistic effect. As expected, a dosedependent reduction in EGFR mRNA expression was observed in the PC-9 cells treated with gefitinib (Fig. 5).

\section{Discussion}

The majority of patients with metastatic NSCLC have a poor outcome, with a median survival time of less than ten months (14). The development of EGFR-TKIs has significantly affected the treatment of NSCLC. EGFR-TKIs, such as gefitinib and erlotinib, have been found to be efficient in the treatment of certain NSCLC patients with EGFR mutations $(7,8)$. However, resistance to TKIs has been found in some NSCLC patients with EGFR mutations. Furthermore, most primary NSCLC cell lines, including cell lines containing wild-type EGFR, are resistant to EGFR-TKI treatment. The exact reasons for this remain unknown. Further investigation in this area would unveil new information and may enhance the therapeutic efficacy and survival of patients with NSCLC. Our study showed that the NSCLC cell line, H1650, which contains a EGFR mutation (del E746-A750), was less sensitive to EGFR-TKIs (15). Since lung cancer cells rapidly develop drug resistance to single TKI therapy, studies on other potential agents that can complement and enhance the anti-neoplastic activity of TKIs are required. Previously, promoter hypermethylation has been recognized as a potential mechanism by which genes regulating cellular proliferation are silenced during cancer development $(16,17)$. Promoter hypermethylation involves DNA methylation of $\mathrm{CpG}$ islands in or near the promoter region of certain genes, rendering them transcriptionally silent. In this study, we demonstrate that the expression level of the EGFR gene is low in the H1650 and H1299 cells. To elucidate the mechanism of the downregulation of EGFR gene expression, we focused on examining the promoter methylation of the EGFR gene in lung cancer cells.

In this study, we demonstrate that the promoter of the EGFR gene is methylated in the H1650 and H1299 cells, which correlates with the downregulation of EGFR gene expression. Of note, 5-aza-CdR, a demethylating agent, increased EGFR gene transcription. This suggests that epigenetic regulation may be responsible for controlling EGF-R gene expression. The downregulation of expression of important cellular growth control genes, such as Ras-association domain family member 1 (RASSF1A) and hypoxia-inducible factor-1 (HIF-1), has been shown to play an important role in cancer progression and metastasis, resulting in a poor outcome that is associated with the promoter hypermethylation of these genes $(18,19)$.

DNA methylation and the associated silencing have been shown to be involved in the development of drug resistance (20), which prompted investigations for the use of a hypomethylation approach to re-sensitize malignant cells to classical cytotoxic agents. 5-Aza-CdR, a potent DNA methylation inhibitor, has exhibited potent anti-neoplastic activity in animal models and has shown promising anticancer activity in hematological malignancies (21), as well as lung cancer (22). Since 5-aza-CdR reactivates genes through blockade of DNA methylation, it has an important therapeutic potential in inhibiting tumorigenesis. This led us to investigate the potential mechanisms of action of the therapeutic agents in malignant tumors, including lung cancer. Montero et al (23) showed that 5-aza-CdR treatment increased the sensitivity to gefitinib, an EGFR-TKI, in EGFRmethylated breast cancer cells. Other published data has suggested that folic acid inhibits the constitutive and induced the activity of the EGFR promoter in colon cancer cells through methylation, as this effect was reversed by 5 -aza-CdR $(24,25)$. 
In a study by Momparler and Ayoub (26), it was observed that patients with stage IV NSCLC who received five cycles of 5-aza-CdR treatment survived 81 months. Consistent with these data, in this study, by evaluating the anti-neoplastic effects of 5-aza-CdR and gefitinib, we showed that a combination of lower doses $\left(<\mathrm{IC}_{50}\right.$ values) of 5-aza-CdR and gefitinib significantly inhibited NSCLC cell growth. Similarly, 5-azaCdR further increased the induction of apoptosis by gefitinib in NSCLC cells. These results suggest that NSCLC cells with EGFR methylation are resistant to gefitinib, and that 5-aza-CdR, a hypomethylating drug, may increase the cellular sensitivity to gefitinib in controlling NSCLC cell growth and apoptosis. The PC-9 cells, which did not harbor a CpG island methylation within the EGFR promoter, were more sensitive to gefitinib than the $\mathrm{H} 1650$ and $\mathrm{H} 1299$ cells. Thus, 5-aza-CdR had no further effect on PC-9 cell proliferation.

Promoter methylation of the EGFR gene is one of the key mechanisms that affects cancer cell sensitivity to TKIs. Previous studies have shown that certain somatic mutations within the TK and ATP-binding domain of the EGFR gene are associated with a response to EGFR-TKIs in NSCLC $(27,28)$. The positive association between EGFR mutations and response to erlotinib or gefitinib in NSCLC patients has been shown in several clinical studies, with a significant enhancement of patient survival (29). However, in larger randomized studies, such as the BR.21 trial, a similar survival advantage was observed for patients treated with erlotinib, independent of EGFR mutations or wild-type EGFR gene, indicating that EGFR mutations were not the only biomarker for predicting NSCLC survival with small-molecule EGFR-TKI treatment (30). Our results suggested that the $\mathrm{CpG}$ island methylation status in the EGFR promoter influenced the sensitivity to gefitinib in NSCLC cells.

Our results demonstrated the enhanced effects of 5-aza-CdR on EGFR-TKI-induced cell growth inhibition and the induction of apoptosis. The reduced expression of EGFR may be due to hypermethylation. In addition, 5-aza-CdR treatment resulted in $\mathrm{CpG}$ island demethylation in the promoter of the EGFR gene. These results suggest that demethylation potentially induces EGFR gene expression. Whether this could affect the sensitivity and therapeutic efficacy of gefitinib remains unknown. In general, methylation causes gene silencing and demethylation induces gene expression. EGFR expression is not considered as a significant predictive factor for a response to gefitinib (31). There is no clear correlation between EGFR expression and gefitinib sensitivity. We reasoned that the demethylation effect of 5-aza-CdR caused the induction of EGFR gene expression. Thus, the balance between methylation and demethylation changes may contribute to the synergistic effects of this combination treatment. On the basis of EGFR demethylation, this would enhance the therapeutic response of gefitinib in NSCLC cells. Further mechanistic studies are required to confirm these results.

Two major EGFR-TKI-resistance mechanisms have been revealed. Gefitinib is an ATP competitive inhibitor of EGFR-TKI. It has been found that the T790M secondary mutation increases the affinity of the oncogenic mutant EGFR for ATP, and this leads to the reduced efficacy of EGFR-TKIs (31). Almost half of the patients with acquired resistance appear to have this mutation $(32,33)$. Met proto-oncogene (MET) amplification is another mechanism that escapes the antitumor effect of EGFR-TKIs, which allows cancer cell survival by persistent enhancement of Akt signaling and MET amplification when the EGFR signal is blocked in the presence of EGFR-TKIs. MET amplification has been found in approximately $20 \%$ of patients with acquired resistance $(34,35)$. The link between MET amplification and promoter methylation of the EGFR gene involving EGFR-TKI resistance has not been reported; whether 5-aza-CdR has any direct or indirect effect on ATP or MET amplification needs to be determined. A recent study showed that 5-aza-CdR decreased c-Met protein expression in NSCLC cells (36), suggesting a potentially novel mechanism of this agent in controlling cancer cell growth.

In conclusion, the data presented in this study show that NSCLC cells with EGFR methylation are more resistant to gefitinib, and that the combination treatment with 5-aza-CdR, a demethylating agent, increases the sensitivity to gefitinib. These results suggest that the combination of a demethylating agent with an EGFR-TKI may have a synergistic anti-lung cancer effect and that the blockade of DNA methylation of the EGFR gene promoter region should be considered as one of the potential mechanisms for reversing resistance to EGFR-TKIs in NSCLC cells. Further studies to better define the role of 5-aza-CdR in controlling cancer cell growth and the potential synergistic antitumor effects of TKIs and 5-aza-CdR in NSCLC are warranted.

\section{References}

1. Ahmed SM and Salgia R: Epidermal growth factor receptor mutations and susceptibility to targeted therapy in lung cancer. Respirology 11: 687-692, 2006

2. Hynes NE and Lane HA: ERBB Receptors and cancer: The complexity of targeted inhibitors. Nat Rev Cancer 5: 341-354, 2005.

3. Yarden Y and Sliwkowski MX: Untangling the ErbB signaling network. Nat Rev Mol Cell Biol 2: 127-137, 2001

4. Citri A, Skaria KB and Yarden Y: The deaf and the dumb: The biology of ErbB-2 and ErbB-3. Exp Cell Res 284: 54-65, 2003.

5. Baselga J: Why the epidermal growth factor receptor? The rationale for cancer therapy. Oncologist 7: 2-8, 2002.

6. Cappuzzo F, Finocchiaro G, Metro G, Bartolini S, Magrini E, Cancellieri A, Trisolini R, Castaldini L, Tallini G and Crino L: Clinical experience with gefitinib: An update. Crit Rev Oncol Hematol 24:31-45, 2006.

7. Haber DA, Bell DW, Sordella R, et al: Molecular targeted therapy of lung cancer: EGFR mutations and response to EGFR inhibitors. Cold Spring Harb Symp Quant Biol 70: 419-426, 2005.

8. Janne PA and Johnson BE: Effect of epidermal growth factor receptor tyrosine kinase domain mutations on the outcome of patients with non-small cell lung cancer treated with epidermal growth factor receptor tyrosine kinase inhibitors. Clin Cancer Res 12: 4416-4420, 2006.

9. Esteller M: Cancer epigenomics: DNA methylation and histonemodification maps. Nat Rev Gene 8: 286-298, 2007.

10. Sharma S, Kelly TK and Jones PA: Epigenetics in Cancer. Carcinogenesis 31: 27-36, 2010.

11. Silva M, Azenha D, Pereira C, et al: Gastric carcionoma and chronic gastritis: epigenetic regulation of CDH1 (E-cadherin), CDKN2A (p16INK4A), PTGS2 (COX-2) and EGFR genes through methylation. Acta Med Port 23: 5-14, 2010 (In Portuguese).

12. Wang Z, Xu J, Geng X and Zhang W: Analysis of DNA methylation status of the promoter of human telomerase reverse transciptase in gastric carcinogenesis. Arch Med Res 41: 1-6, 2010.

13. Taron M, Rosell R, Felip E, et al: BRCA1 mRNA expression levels as an indicator of chemoresistance in lung cancer. Hum Mol Genet 13: 2443-2449, 2004.

14. Grivaux M,Zureik M, Marsal L, et al: Five-year survival for lung cancer patients managed in general hospitals. Rev Mal Respir 28: e31-e38, 2011. 
15. Gadgeel SM, Ali S, Philip PA, Wozniak A and Sarkar FH: Genistein enhances the effect of epidermal growth factor receptor tyrosine kinase inhibitors and inhibits nuclear factor kappa B in non-small cell lung cancer cell lines. Cancer 115: 2165-2176, 2009.

16. Vucic EA, Brown CJ and Lam WL: Epigenetics of cancer progression. Pharmacogenomics 9: 215-234, 2008.

17. Duffy MJ, Napieralski R, Martens JW, et al: Methylated genes as new cancer biomarkers. Eur J Cancer 45: 335-346, 2009.

18. Kim YT, Park SJ, Lee SH, et al: Prognostic implication of aberrant promoter hypermethylation of $\mathrm{CpG}$ islands in adenocarcinoma of the lung. J Thorac Cardiovasc Surg 130: 1378, 2005.

19. Safar AM, Spencer H III, Su X, et al: Methylation profiling of archived non-small cell lung cancer: a promising prognostic system. Clin Cancer Res 11: 4400-4405, 2005.

20. Plimack ER, Stewart DJ and Issa JP: Combining epigenetic and cytotoxic therapy in the treatment of solid tumors. J Clin Oncol 25: 4519-4521, 2007.

21. Daskalakis M, Blaqitko-Dorfs N and Hackanson B: Decitabine. Recent Results Cancer Res 184: 131-157, 2010.

22. Chai G, Li L, Zhou W, et al: HDAC inhibitors act with 5-aza2'-deoxycytidine to inhibit cell proliferation by suppressing removal of incorporated abases in lung cancer cells. PLoS One 3 . e2445, 2008

23. Montero AJ, Díaz-Montero CM, Mao L, et al: Epigenetic inactivation of EGFR by $\mathrm{CpG}$ island hypermethylation in cancer. Cancer Biol Ther 5: 1494-1501, 2006.

24. Majumdar AP, Kodali U and Jaszewski R: Chemopreventive role of folic acid in colorectal cancer. Front Biosci 9: 2725-2732, 2004.

25. Nagothu KK, Rishi AK, Jaszewski R, Kucuk O and Majumdar AP Folic acid-mediated inhibition of serum-induced activation of EGFR promoter in colon cancer cells. Am J Physiol Gastrointest Liver Physiol 287: G541-G546, 2004.

26. Momparler RL and Ayoub J: Potential of 5-aza-2'-deoxycytidine (Decitabine) a potent inhibitor of DNA methylation for therapy of advanced non-small cell lung cancer. Lung Cancer 34 (Suppl 4): S111-S115, 2001
27. Lynch TJ, Bell DW, Sordella R, et al: Activating mutations in the epidermal growth factor receptor underlying responsiveness of non-small-cell lung cancer to gefitinib. N Engl J Med 350: 2129-2139, 2004

28. Paez JG, Jänne PA, Lee JC, et al: EGFR mutations in lung cancer: correlation with clinical response to gefitinib therapy. Science 304: 1497-1500, 2004.

29. Sharma SV, Bell DW, Settleman J and Haber DA: Epidermal growth factor receptor mutations in lung cancer. Nat Rev Cancer 7: 169-181, 2007.

30. Tsao M-S, Sakurada A, Cutz J-C, et al: Erlotinib in lung cancer molecular and clinical predictors of outcome. N Engl J Med 353: 133-144, 2005.

31. Yun CH, Menqwasser KE, Toms AV, et al: The T790M mutation in EGFR kinase causes drug resistance by increasing the affinity for ATP. Proc Natl Acad Sci USA 105: 2070-2075, 2008.

32. Pao W, Miller VA, Politi KA, et al: Acquired resistance of lung adenocarcinomas to gefitinib or erlotinib is associated with a second mutation in the EGFR kinase domain. PLoS Med 2: e73, 2005.

33. Kosaka T, Yatabe Y, Endoh H, et al: Analysis of epidermal growth factor receptor gene mutation in patients with non-small cell lung cancer and acquired resistance to gefitinib. Clin Cancer Res 12: 5764-5769, 2006.

34. Engelman JA, Zejnullahu K, Mitsudomi T, et al: MET amplification leads to gefitinib resistance in lung cancer by activating ERBB3 signaling. Science 316: 1039-1043, 2007.

35. Guo A, Villen J, Kornhauser J, et al: Signaling network assembled by oncogenic EGFR and c-met. Proc Natl Acad Sci USA 105: 692-697, 2008.

36. Watanabe K, Emoto N, Hamano E, et al: Genome structurebased screening identified epigenetically silenced microRNA associated with invasiveness in non-small-cell lung cancer. Int J Cancer 130: 2580-2590, 2012. 